1-cm cell of the sample solution. The filtered fluorescent light was observed photoelectrically at right angles to the incident beam. Lifetimes were also measured photoelectrically using a flash apparatus of $10 \mu \mathrm{sec}$ resolving time. To ensure that effects observed were not caused by differences in optical density, the spectrum of each solution was recorded using a Cary Model 14 spectrophotometer. The optical densities of corresponding solutions in heavy water and ordinary water were the same within our experimental error.

The results of intensity measurements are given in Table I for several salts as $I_{\mathrm{D}} / I_{\mathrm{H}}$, the ratio of the intensity of emission of the salt in deuterated water $\left(I_{\mathrm{D}}\right)$ to the intensity in protonated water $\left(I_{\mathrm{H}}\right)$. Lifetimes were measured for the terbium salts and are recorded as $\tau_{\mathrm{D}}, \tau_{\mathrm{H}}$ and $\tau_{\mathrm{D}} / \tau_{\mathrm{H}}$, the subscripts $\mathrm{D}$ and $\mathrm{H}$ denoting lifetime in heavy water and in ordinary water, respectively. Values of the intensity ratio and lifetime are both reproducible to an accuracy of about $\pm 10 \%$. The lifetimes follow a simple exponential decay in all of the terbium salts studied. Our values for $\mathrm{TbCl}_{3}$ in $\mathrm{H}_{2} \mathrm{O}\left(4.7 \times 10^{-4} \mathrm{sec}\right)$ and $\mathrm{Tb}_{2}\left(\mathrm{SO}_{4}\right)_{3}$ in $\mathrm{H}_{2} \mathrm{SO}_{4}\left(1.4 \times 10^{-3}\right.$ sec) show good agreement with those of Kondrat'eva ${ }^{8}$ who obtained $5.5 \times 10^{-4} \mathrm{sec}$ and $1.5 \times 10^{-3} \mathrm{sec}$, respectively.

The substitution of deuterated water for ordinary protonated water markedly increases the light yield in most cases. The effect seems to be inversely dependent upon the energy gap between the highest level of the ground multiplet and the resonance level of the rareearth ion from which emission occurs. Thus, the order of magnitude of change of emission intensity is $\mathrm{Eu}^{3+}>$ $\mathrm{Tb}^{3+}>\mathrm{Gd}^{3+}$, while the energy gaps between the resonance level and the high-lying ground level for the same ions are 12300,14200 , and $32100 \mathrm{~cm}^{-1}$, respectively. On this basis it is easy to explain why $\mathrm{GdCl}_{3}$ shows no increase of intensity when it is dissolved in deuterated water. The large energy gap between the resonance level and the ground level already renders radiationless transitions very unlikely even when the coordinating group is $\mathrm{O}-\mathrm{H}$. Thus, substitution of $\mathrm{O}-\mathrm{D}$ causes no further detectable effect. We have not yet accounted for the differences in the ratios $I_{\mathrm{D}} / I_{\mathbf{H}}$ and $\tau_{\mathrm{D}} / \tau_{\mathrm{H}}$. One might expect these ratios to be similar. The differences are definitely greater than our experimental error. Further experiments are in progress to determine the cause of these differences.

We plan to extend our measurements to other rareearth salts and also to investigate the effect of deuterating such other common solvents as methanol. We also intend to study the properties of deuterated chelates, both in ordinary and in deuterated solvents.

The above results lead us to believe that chelates under conditions of complete deuteration should have significantly higher quantum yields than ordinary protonated chelates. In addition, deuteration of the solvent may also be important, since solvent interaction is an important factor in chelate emission. ${ }^{2}$ Enhance- ment of fluorescence efficiencies by deuteration, as reported here, may make it possible to use rare-earth chelates as optical masers even at room temperature.

We are grateful to Professor G. A. Crosby for calling our attention to the possibility of a deuterium effect in rare-earth chelates, to Mr. C. C. Stanley for the measurement of lifetimes and to Mr. C. D. Krull for assistance in the preparation of the experimental samples.

\footnotetext{
* Supported in part by Space Systems Division, U. S. Air Force Systems Command, Los Angeles, California, under Contract AF (609)-1457.

1 A. Lempicki and H. Samelson, Appl. Phys. Letters 4, 133 (1963).

2 A. Yariv and J. P. Gordon, Proc. IEEE 51, 4 (1963).

${ }^{3}$ W. R. Dawson, J. L. Kropp, and M. W. Windsor, Symposium on Molecular Structure and Molecular Spectroscopy, Columbus, Ohio, 1963.

${ }^{4}$ M. R. Wright, R. P. Frosch, and G. W. Robinson, J. Chem. Phys. 33, 934 (1960).

${ }_{5}$ C. A. Hutchinson and B. W. Magnum, J. Chem. Phys. 32, $1261(1960)$.

${ }^{6}$ G. W. Robinson, J. Mol. Spectry. 6, 58 (1961).

${ }^{8}$ E. V. Sayre, D. G. Miller, and S. Freed, J. Chem. Phys. 26, 109 (1957).

${ }^{8}$ E. V. Kondrat'eva, Opt. Spectry. 8, 66 (1960).
}

\section{Comments and Errata}

\section{Interpretation of Hot-Atom Data*}

\author{
Chi-hua Hsiung and Adon A. Gordus \\ Department of Chemistry, University of Michigan, \\ Ann Arbor, Michigan \\ (Received 27 June 1963)
}

$\mathbf{R}$ ECENTLY, Root and Rowland ${ }^{1}$ determined the yields $Y$ of $\mathrm{DT}, \mathrm{CH}_{3} \mathrm{~T}$, and $\mathrm{HT}$ in a mixture of $\mathrm{D}_{2}$ and $\mathrm{CH}_{4}$ with 3 -mole $\% \mathrm{O}_{2}$ scavenger. The tritium was generated by the $\mathrm{He}^{3}(n, p) \mathrm{T}^{3}$ process. They noted that $Y_{\mathrm{DT}} / X_{\mathrm{D}_{2}}$ and $Y_{\mathrm{CH}_{3} \mathrm{~T}} / X_{\mathrm{CH}_{4}}$ both decreased as $X_{\mathrm{CH}_{4}}$ increased. This was interpreted by them as an indication that the tritium hot atom loses more energy on the average when colliding with $\mathrm{CH}_{4}$ than with $\mathrm{D}_{2}$, and with increasing $\mathrm{CH}_{4}$ has less of an opportunity to undergo a reactive collision before becoming thermalized.

There are three main effects which appear to determine the yields of gas-phase hot-atom chemical reactions. They are: (1) the collisional cross section, (2) the reaction probability per collision, and (3) the average energy lost by the hot atom per nonreactive collision. All three undoubtedly vary with the energy of the hot atom.

It would seem that before an attempt is made to determine the second or third effect, it is necessary to examine data in terms of the collision probability rather than in terms of the mole fraction.

If it is assumed that the various collision cross sections are energy-independent, or if energy-averaged 
TABLE I. Yields per collision probability. ${ }^{\mathrm{a}}$

\begin{tabular}{cccc}
\hline$X_{\mathrm{CH} 4}$ & $f_{\mathrm{CH} 4}$ & $Y_{\mathrm{DT}} / f_{\mathrm{D}_{2}}$ & $Y_{\mathrm{CH} 3 \mathrm{~T}} / f_{\mathrm{CH}_{4}}$ \\
\hline 0.056 & 0.086 & 1120 & 349 \\
0.101 & 0.152 & 1150 & 322 \\
0.174 & 0.251 & 1130 & 331 \\
0.270 & 0.370 & 1150 & 349 \\
0.379 & 0.492 & 1170 & 356 \\
0.464 & 0.578 & 1190 & 339 \\
0.684 & 0.772 & 1180 & 343 \\
0.866 & 0.906 & 1240 & 337 \\
\hline
\end{tabular}

a The presence of $\mathrm{O}_{2}$ was taken into account.

collision cross sections are used, then the probability of collision of tritium with Molecule $i, f_{i}$, in a mixture of $A, B, C, \cdots, i$, will be ${ }^{2}$

$$
f_{\mathrm{i}}=\mathrm{X}_{\mathrm{i}} \sigma_{\mathrm{i}, \mathrm{T}} / \sum X_{\mathrm{i}} \sigma_{\mathrm{i}, \mathrm{T}}
$$

where $X_{\mathrm{i}}$ is the mole fraction of $\mathrm{i}, \sigma_{\mathbf{i}, \mathbf{T}}$ is the collision cross section and $=\pi\left(r_{\mathrm{i}}+r_{\mathrm{T}}\right)^{2}$, and $r$ is the collision radius. Thus $\sum f_{\mathrm{i}}=1$.

Using collision radii determined at thermal energies ${ }^{3}$ $\left(\mathrm{T}=1.2, \mathrm{CH}_{4}=2.1, \mathrm{D}_{2}=1.4, \mathrm{O}_{2}=1.8 \AA\right.$ ) we evaluated the data of Root and Rowland ${ }^{1}$ in terms of collision probability. From Table $I$ it is seen that as $X_{\mathrm{CH}_{4}}$ increases, $Y_{\mathrm{DT}} / f_{\mathrm{D}_{2}}$ appears to increase, and $Y_{\mathrm{CH}_{3} \mathrm{~T}} / f_{\mathrm{CH}_{4}}$ appears constant.

If the data of Table $I$ are accepted and the reasoning given by Root and Rowland ${ }^{1}$ is used then the conclusion would be that $D_{2}$ absorbs energy more effectively than $\mathrm{CH}_{4}$; this conclusion is opposite to that proposed by them.

It may be equally valid to try to explain the original data in terms of reaction probability effects. Using neutron cooling-down theory we have calculated yields for reactions of a hot atom with mixtures of two molecules $\mathrm{A}$ and $\mathrm{B} .{ }^{4}$ Molecule $\mathrm{A}$ was considered the more effective moderator. Depending on the values assigned to the reaction probability integrals, ${ }^{2} I$, the relative displacements and shapes of the reaction probability curves, and the moderation efficiencies, we can obtain data for $Y_{\mathrm{A}} / X_{\mathrm{A}}$ and $Y_{\mathrm{B}} / X_{\mathrm{B}}$ which either increase, decrease, or remain constant with increasing $X_{\mathrm{B}}$.

Because of the interrelationships between the three effects in governing the yields and the current lack of knowledge about the energy dependence of the effects, it appears impossible, unless a number of additional assumptions are made, to use these hot-atom data to conclude which of the molecules, $\mathrm{D}_{2}$ or $\mathrm{CH}_{4}$, is the more efficient tritium moderator.

* Supported in part by the U. S. Atomic Energy Commission, Division of Research.

1 J. W. Root and F. S. Rowland, J. Chem. Phys. 38, 2030 (1963).

2 P. J. Estrup and R. Wolfgang, J. Am. Chem. Soc. 82, 2665 (1960).

3 S. Chapman and T. G. Cowling, The Mathematical Theory of Non-Uniform Gases (Cambridge University Press, New York, 1952) p. 229.

${ }^{4}$ Consider $\mathrm{A}=\mathrm{D}_{2}$ and $\mathrm{B}=\mathrm{CH}_{4}$.

\section{Notes}

\section{Approximate Quantum-Mechanical Law of Corresponding States}

\author{
IRWIN OpPenheIM \\ Department of Chemistry, Massachusetts Institute of Technology
}

(Received 5 June 1963)

THE theory of the statistical thermodynamics of quantum fluids developed by Kirkwood and $\mathrm{Mazo}^{1-3}$ and by Oppenheim ${ }^{4}$ leads to expressions for the internal energy and the pressure of the form

$$
\begin{array}{r}
E(N, v, T)=E=\frac{3}{2} N k \tau+\frac{1}{2} v \int n_{c}^{(2)}(R, \tau) U(R) d \mathbf{R} \\
=E_{c}(N, v, \tau), \\
\begin{array}{r}
p(v, T)=p=(k \tau / v)-\frac{1}{6} \int n_{c}{ }^{(2)}(R, \tau) \mathbf{R} \cdot \nabla_{R} U d \mathbf{R} \\
=p_{c}(v, \tau),
\end{array}
\end{array}
$$

where $N$ is the number of particles in the system, $k$ is Boltzmann's constant, $v$ is the volume per particle, $U(R)$ is the intermolecular pair potential, $n_{c}^{(2)}$ is the classical generic pair distribution function which is a function of $R$, the scalar distance between the particles, $v$, and the parameter $\tau=\tau(v, T)$, the effective temperature which is defined by the fact that $3 k \tau / 2$ is the mean kinetic energy per particle. In a classical system, $\tau$ is the thermodynamic temperature $T$. The quantity $E_{c}(N, v, \tau)$ is the classical internal energy of the system with $N$ particles, volume per particle $v$, and effective temperature $\tau$. Similarly $p_{c}$ is the classical pressure for such a system. Equations (1) and (2) apply to equilibrium systems of spherically symmetric molecules and are derived by making use of the superposition approximation in triplet space and assumptions concerning the nature of averages of the dyad $\mathbf{p}_{i} \mathbf{p}_{j}\left(\mathbf{p}_{i}\right.$ is the vector momentum of particle $i$ ) over all momentum space and over the configuration spaces of $N, N-1, N-2$, and $N-3$ particles.

The approximate quantum-mechanical theory which leads to Eqs. (1) and (2) predicts a simple form for the quantum-mechanical law of corresponding states. We predict that systems composed of similar particles have the same energy and pressure for the same value of the reduced volume, $v^{*}=v / a^{3}$, and the reduced effective temperature, $\tau^{*}=k \tau / \epsilon$. The parameter $a$ is a characteristic length and $\epsilon$ is a characteristic energy of the intermolecular force. That is,

$$
\begin{aligned}
& \epsilon_{\mathrm{II}} E_{\mathrm{I}}\left(N, v^{*}, T_{\mathrm{I}}^{*}\right)=E_{\mathrm{II}}\left(N, v^{*}, T_{\mathrm{II}}^{*}\right)_{\epsilon}, \\
& \left(a^{3} / \epsilon\right)_{\mathrm{I}} p_{\mathrm{I}}\left(v^{*}, T_{\mathrm{I}}^{*}\right)=p_{\mathrm{II}}\left(v^{*}, T_{\mathrm{II}}^{*}\right)\left(a^{3} / \epsilon\right)_{\mathrm{II}},
\end{aligned}
$$

when $T_{\mathbf{I}}{ }^{*}$ and $T_{\mathbf{I I}}{ }^{*}$ are chosen such that

$$
\tau_{\mathrm{I}}^{*}\left(\vartheta^{*}, T_{\mathrm{I}}^{*}\right)=\tau_{\mathrm{II}}^{*}\left(\nu^{*}, T_{\mathrm{II}}^{*}\right) \text {. }
$$

